\title{
Modification of cotton fabrics with 2-diethylaminoethyl chloride for salt-free dyeing with anionic dyes
}

\author{
Peixin Tang • Leilah-Marie E. Lockett $\cdot$ Mengxiao Zhang • Gang Sun $\mathbb{D}$
}

Received: 4 March 2021 / Accepted: 11 May 2021/Published online: 21 May 2021

(C) The Author(s) 2021

\begin{abstract}
A chemical modification of cotton fabrics by 2 -diethylaminoethyl chloride (DEAE-Cl) was achieved, and the resulted cotton fabrics demonstrated salt-free dyeing properties with anionic dyes. Nucleophilic property of hydroxyl groups in cotton cellulose was enhanced under alkaline conditions and could react with $\mathrm{DEAE}-\mathrm{Cl}$, a chemical possessing both nucleophilic and electrophilic sites. The monolayered DEAE-grafted cotton cellulose could further react with DEAE-Cl to form multiple cationic quaternary ammonium salts (denoted as DEAE@Cotton), which are highly interactive with anionic dye molecules. The strong electrostatic interactions between the DEAE@Cotton and the dyes eliminated the use of inorganic salts in cotton dyeing process. The chemical
\end{abstract}

structure and property of DEAE@Cotton were characterized and compared with untreated cotton. The DEAE@Cotton can be dyed in a salt-free system, and the dye exhaustion was faster than the conventional dyeing method due to the robust electrostatic interactions of the fabrics with anionic dyes. The dyed fabrics demonstrated outstanding color fastness under repeated washing, light exposure, and crocking. The dye adsorption process on DEAE@Cotton follows Langmuir isotherm model $\left(\mathrm{R}^{2}=0.9667\right)$. The mechanism of enhanced dyeability was experimentally proved by treating the fabric with other anionic dyes in a salt-free system, proving the process to be environmentally friendly and cost-effective.

P. Tang · L.-M. E. Lockett · M. Zhang · G. Sun ( $\square)$ Department of Biological and Agricultural Engineering, University of California, Davis, CA 95616, USA

e-mail: gysun@ucdavis.edu 


\section{Graphic abstract}
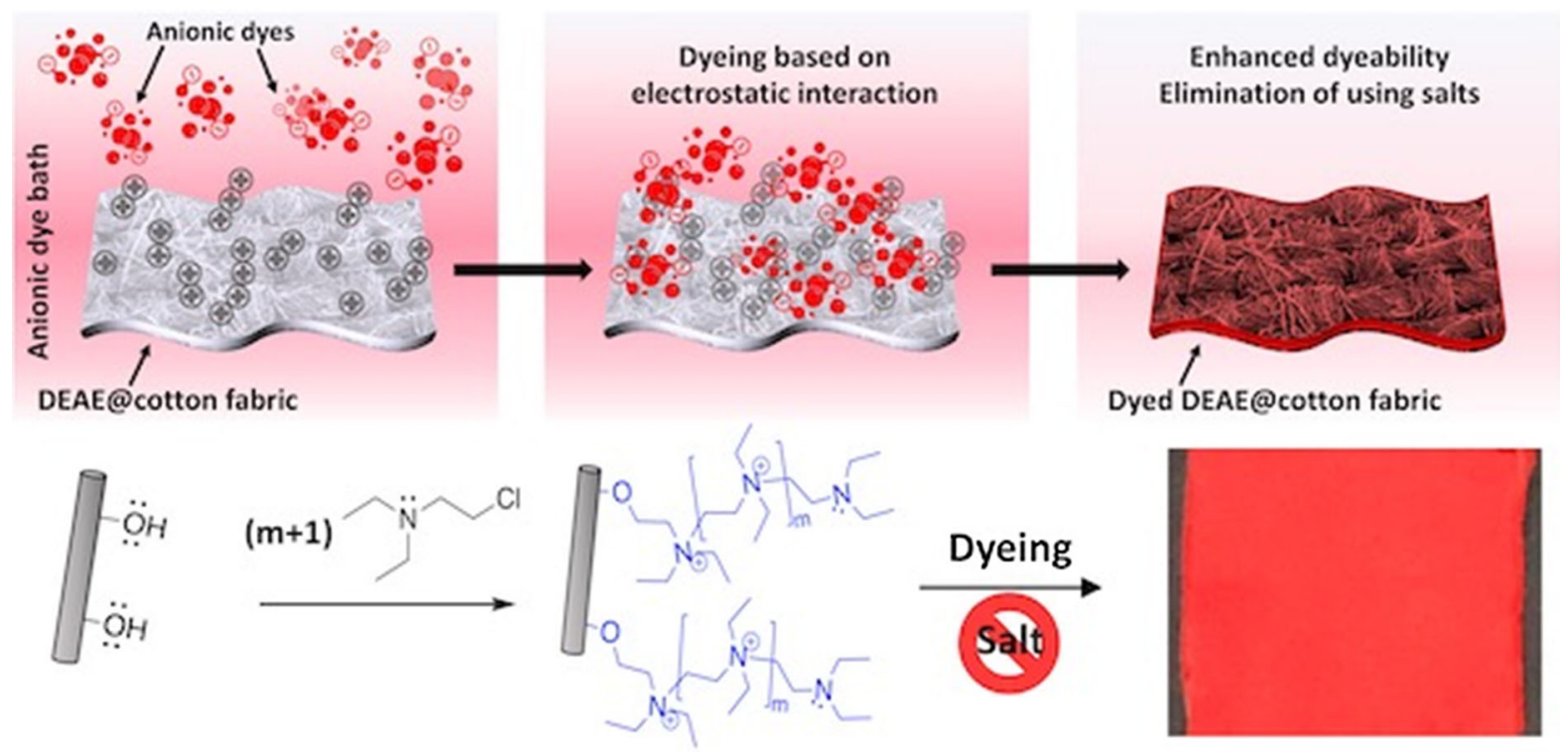

Keywords Cationization - Cellulose ·

Environmentally friendly dyeing $\cdot$ Direct dye $\cdot$ Saltfree

\section{Introduction}

Cotton is the most popular fiber used in apparels because of its hydrophilicity, comfort, and softness. Coloration of cotton textiles is an indispensable need. However, the colorations of the cotton products, especially reactive and direct dyeing processes, are major sources of water pollutions. Residual dyes, inorganic salts, and additives account for the major pollutants in the discharged wastewater. The use of salts and chemicals for dyeing cotton is necessary to achieve desired color depth and color fastness of the fabrics (Dixit et al. 2015; Kant 2012). Specifically, a large quantity of inorganic salts is commonly used in dyeing cotton fabrics with direct and reactive dyes. Even by increasing salts in the dyeing baths, residual dyes still unavoidably exist in the dyeing wastewater. The used inorganic salts in the dye effluents are not easy to be recycled, resulting in concerns to environmental health and safety (Khatri et al. 2015). Thus, many approaches have been made to reduce residual dyes or eliminate the use of salts in the dyeing processes, including: (1) synthesis of dyes with improved affinity or reactivity toward cotton cellulose (Wang et al. 2016; Xiao et al. 2017; Zhang and Zhang 2015; Zheng et al. 2012); (2) modification of cotton cellulose by introducing stronger dye interactive groups (Arivithamani and Giri Dev, 2017; Fang et al. 2017; Fang et al. 2019; Hauser and Tabba 2001; Ma et al. 2016; Ma et al. 2017; SadeghiKiakhani and Safapour 2018; Samanta et al. 2015; Samanta et al. 2016; Toprak et al. 2018); and (3) varying the solvent system of dyeing (Dong et al. 2019; Mu et al. 2019; Wang et al. 2018; Xia et al. 2018; Zhao et al. 2018).

Hydrated cotton cellulose contains negative charges on surfaces in the water system, therefore, anionic dyes such as direct and reactive dyes are mostly repulsed electrostatically by the fibers, requiring the use of electrolytes (i.e., inorganic salts) in assisting dye exhaustion. Therefore, any approach that can diminish the charge repulsion between cotton and dyes could reduce the use of the salts in the dyeing bath, which has triggered the practices of synthesis of new dyes (Zheng et al. 2012) and cationic modifications of cotton fibers (Farrell et al. 2014, 2017; Fu et al. 2013; Hashem et al. 2003; Hauser and Tabba 2001). 
Herein, we introduce a new strategy to modify cotton fabrics with 2-diethylaminoethyl chloride (DEAE-Cl) through a unique reaction consisted of a nucleophilic substitution followed by a self-propagation of DEAE-Cl. Relying on the coexistence of nucleophilic and electrophilic sites in DEAE-Cl, four reaction paths could occur during the modification (Scheme 1): (1) DEAE-Cl directly reacts with alkaline-activated cotton fabrics to form a monolayer of tertiary amines on cotton; (2) DEAE-Cl undergoes self-propagation and stays in the modification solution; (3) the self-propagated DEAE-Cl reacts with alkaline-activated cotton fabrics; and (4) DEAE-Cl self-propagated on alkaline-activated cotton to generate multilayered quaternary ammonium salts.

The achievement of a high density of cationic quaternary ammonium salts on DEAE@Cotton makes the dyeing performance less sensitive to $\mathrm{pH}$ changes, which is different from the cotton fabrics modified with amines (Zhang et al. 2007; Zhu et al. 2017). The chemical structures of DEAE@Cotton fabrics were characterized with scanning electron microscope (SEM), Fourier-transform infrared spectroscopy (FTIR), thermogravimetric analysis (TGA), and Zeta potentials. The optimal dyeing performances of the DEAE@Cotton were achieved by carefully selecting different modification parameters, and the mechanical strength, color intensity, and wash fastness of the products were compared with that of the untreated cotton. The lightfastness and crocking fastness of dyed DEAE@Cotton were also evaluated.

\section{Experimental section}

Chemicals and materials

Scoured and bleached woven cotton fabrics (Style \# 400 plain woven weighting $98 \mathrm{~g} / \mathrm{m}^{2}, 60 \times 60$ ), was purchased from TestFabrics Inc. (USA). Sodium hydroxide $(\mathrm{NaOH})$, 2-diethylaminoethyl chloride hydrochloric acid (DEAE-Cl), sodium chloride $(\mathrm{NaCl})$, denatured ethyl alcohol $(\mathrm{EtOH})$, direct red 23 (DR23), direct yellow 27 (DY27), direct blue 71 (DB71), acid red 151 (AR151), and reactive blue 4 (RB4) were purchased from Sigma-Aldrich (USA). All chemicals were directly used as received.

\section{Modification of cotton fabrics with DEAE-Cl}

Pre-washed cotton fabrics were activated in $\mathrm{NaOH} /$ EtOH solution $(120 \mathrm{~g} / \mathrm{L})$ for $30 \mathrm{~min}$ at room
1)

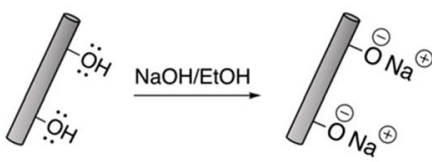

Cellulose
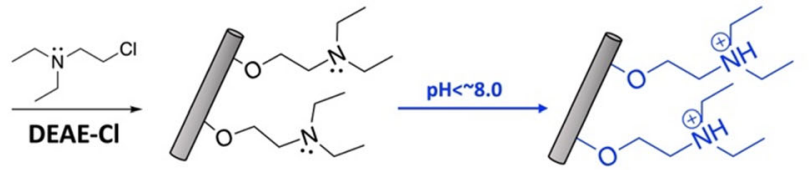

Enhanced dyeability

2)

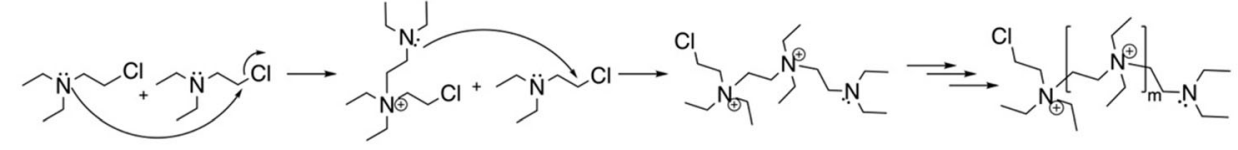

3)

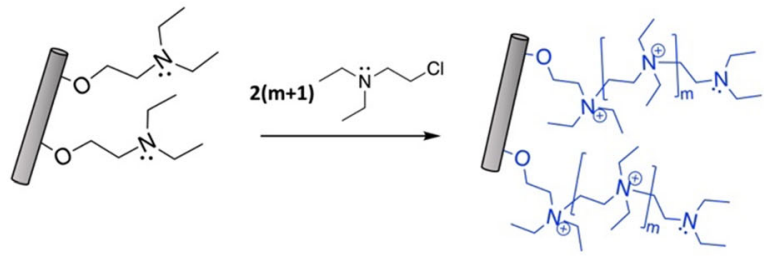

4)
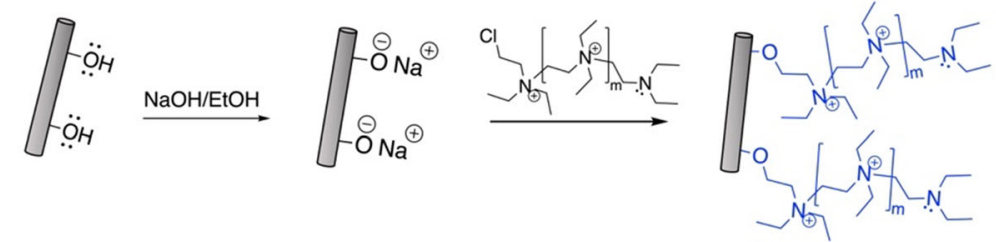

Enhanced

dyeability

Scheme 1 Potential reactions of cotton fabrics with DEAE-Cl under alkaline conditions 
temperature, then the fabrics were transferred into a DEAE-Cl/EtOH solution with a specific DEAE-Cl concentration. The $\mathrm{pH}$ of the final solution was adjusted to around 12.0 using $1 \mathrm{M} \mathrm{NaOH}$ if needed. The fabrics were reacted under specific temperature and time with gentle stirring. After the modification, the DEAE@Cotton fabrics were washed with ethanol (5 $\mathrm{min})$ and water $(5 \mathrm{~min})$ to remove any unreacted chemicals. The DEAE@Cotton was dried at $80{ }^{\circ} \mathrm{C}$ for $5 \mathrm{~min}$ and stored for further use.

\section{Characterization of DEAE@Cotton fabrics}

Fourier-transform infrared spectroscopy (FTIR) of fabric samples was obtained from a Nicolet 6700 FTIR spectrometer (Thermo Electron Co., USA) in absorbance mode at a range of $4000-400 \mathrm{~cm}^{-1}$ with 64 scans and $2 \mathrm{~cm}^{-1}$ resolution. The sample pellets were prepared by mixing $2 \mathrm{mg}$ of fabric powders with $200 \mathrm{mg}$ oven-dried potassium bromide (KBr). Thermogravimetric analysis (TGA) tests were performed to evaluate thermostability of cotton fabrics before and after DEAE-Cl modification. Specifically, fabric powders $(\sim 8 \mathrm{mg})$ were loaded in a TGA-60 system (Shimadzu Science Instruments, Inc., USA) with an aluminum pan. The samples were first heated to $120^{\circ} \mathrm{C}$ and held for 2 min under nitrogen flow $(30 \mathrm{~mL} / \mathrm{min}$ ) to eliminate all free water in the samples. Then, a heating rate of $10{ }^{\circ} \mathrm{C} / \mathrm{min}$ was applied to reheat the samples from 35 to $600{ }^{\circ} \mathrm{C}$ under nitrogen environment $(30 \mathrm{~mL} / \mathrm{min})$. Scanning electron microscope (SEM) images were captured with Quattro ESEM (Thermo Fisher Scientific, USA). The fabrics were coated with a thin layer of gold ( $\sim 120 \AA$ ) by a gold sputter before SEM monitoring. Zeta potentials of untreated cotton and DEAE@Cotton were measured by Zetasizer Nano ZS90 with a DTS 1070 cuvette (Malvern Panalytical, UK). Specifically, the fabric samples were cut into fine powders and disperse in distilled water with $10 \mathrm{~min}$ of sonication (i.e., $100 \mathrm{mg}$ fabric powder in $30 \mathrm{~mL}$ of $\mathrm{H}_{2} \mathrm{O}$ ). Different $\mathrm{pH}$ of the fabric suspension was adjusted by $\mathrm{NaOH}$ or $\mathrm{HCl}$. The freshly prepared fabric suspension was used for zeta potential tests.

The tensile strengths of cotton fabrics before and after DEAE-Cl treatment were tested on an INSTRON 5566 tester (Instron Corporation; MA, USA) according to the American Society for Testing and Materials (ASTM) D5035-06 method. The tensile strength on the filling direction of the fabric was measured to represent the mechanical strength of unmodified cotton (named as Cotton in the following discussion) and DEAE@Cotton. The tensile strength recovery (TSR) was calculated from Eq. (1).

$\mathrm{TSR}=\frac{T S_{i}}{T S_{C}} \times 100 \%$

where $T S_{i}$ is the tensile strength in a unit of Newton (N) of treated fabrics and $T S_{C}$ is the tensile strength in $\mathrm{N}$ of Cotton fabrics.

A wicking test of fabrics was performed according to the American Association of Textile Chemists and Colorists (AATCC) 197 test method. Specifically, fabrics were cut in a size of $165 \mathrm{~mm} \times 25 \mathrm{~mm}$ and conditioned in a conditioning room (temperature $=21{ }^{\circ} \mathrm{C}$, relative humidity $=65 \%$ ) for at least $4 \mathrm{~h}$ before testing. Then, the fabric was vertically hung by immersing the other end of the fabric in a direct red 23 solution $(50 \mathrm{mg} / \mathrm{L})$ with a depth of $5 \mathrm{~mm} \pm 1 \mathrm{~mm}$. After $30 \mathrm{~min}$, the wicking distance was measured based on the length of dye vertically traveled. Each sample was tested in triplicates.

Dyeing fabrics with anionic dyes

Conventional and modified dyeing processes with direct dyes were applied on Cotton and DEAE@Cotton fabrics. Specifically, dye concentration of $2 \%$ owf was used in all dyeing processes. The dyeing baths with a liquor ratio of 50:1 were prepared with or without $5 \mathrm{~g} / \mathrm{L}$ or $10 \mathrm{~g} / \mathrm{L} \mathrm{NaCl}$. The fabrics were first rinsed with deionized water and squeezed before putting into the dyeing bath (pre-heated to $40{ }^{\circ} \mathrm{C}$ ). Then, the temperature of the dyeing bath was increased to $60{ }^{\circ} \mathrm{C}$ within $5 \mathrm{~min}$, and the fabrics were dyed under $60{ }^{\circ} \mathrm{C}$ with stirring for another $35 \mathrm{~min}$. Finally, the dyed fabrics were washed with cold water for three times and dried at $80{ }^{\circ} \mathrm{C}$ for $5 \mathrm{~min}$.

Characterization of dyed fabrics

A GretagMacbeth ColorEye 7000A tester (Akron, Ohio, USA) was used to measure the color coordinates $L^{*}, a^{*}$, and $b^{*}$ and $\mathrm{K} / \mathrm{S}$ values of the fabrics. The color difference $(\Delta \mathrm{E})$ of dyed DEAE@Cotton over the conventionally dyed Cotton was calculated from Eq. (2). The color depth is evaluated by K/S values 
and can be calculated from the reflectance $(R)$ of the fabrics based on Eq. (3).

$$
\Delta E=\sqrt[2]{\left(L_{C}^{*}-L_{D}^{*}\right)^{2}+\left(a_{C}^{*}-a_{D}^{*}\right)^{2}+\left(b_{C}^{*}-b_{D}^{*}\right)^{2}}
$$

where $L^{*}, a^{*}$ and $b^{*}$ refer to the color changing values from light to dark, red to green, and blue to yellow from positive to negative, respectively. The subscripts of $C$ and $D$ stand for the specific values of the conventionally dyed Cotton and the dyed DEAE@Cotton.

$\mathrm{K} / \mathrm{S}=\frac{(1-R)^{2}}{2 R}$

where $\mathrm{K}$ is the absorbance coefficient, and $\mathrm{S}$ is the scattering coefficient.

The wash fastness of Cotton and DEAE@Cotton fabrics after dyeing was measured with washing and drying machines according to standard method of AATCC LP1-2018. The color fastness was evaluated by the K/S values of dyed fabrics after each washing cycle.

The lightfastness of dyed fabrics was evaluated based on the fabric's color change after 3 and $8 \mathrm{~h}$ of daylight exposures. Specifically, the dyed fabric was irradiated in a Spectrolinker ${ }^{\mathrm{TM}}$ XL-1500 UV crosslinker equipped with six cool white bulbs $(6 \times 15 \mathrm{~W})$. The distance between the fabric and the light source was controlled as $15 \mathrm{~cm}$. After irradiation, the color of the fabric was measured by the GretagMacbeth ColorEye $7000 \mathrm{~A}$ tester. The $\Delta \mathrm{E}$ values were calculated from Eq. (2). The crocking fastness of the fabric was evaluated by using an AATCC crockmeter (Atlas Electric Devices Co., IL, USA) based on AATCC Test Method 8. A white cotton fabric was used as the reference to evaluate the color transfer during crocking fastness tests. The tests were performed in duplicates.

\section{Dyeing mechanism and kinetics}

The adsorption of dyes on Cotton and DEAE@Cotton fabrics was studied to fit either Langmuir (Eq. 4) or Freundlich isotherm model (Eq. 5). The fabrics were dyed in the dyeing bath with different initial concentrations (ranging from 10 to $200 \mathrm{mg} / \mathrm{L}$ ) at room temperature $\left(25 \pm 2{ }^{\circ} \mathrm{C}\right)$, and DR23 was selected as the anionic dye representative. When the dyeing process reached the equilibrium $(24 \mathrm{~h})$, the residual dyeing solutions were scanned with a UV-vis spectrophotometer (Evolution 600, Thermo Scientific, USA), and the absorption intensity at the maximum wavelength of $520 \mathrm{~nm}\left(\lambda_{\max }=520 \mathrm{~nm}\right)$ was recorded for the measurements of the dye concentrations in the effluent $\left(C_{e}\right)$ and calculation of the amounts of dyes adsorbed on the cotton fabrics $\left(Q_{e}\right)$.

$Q_{e}=\frac{Q_{m} k_{L} C_{e}}{1+k_{L} C_{e}}$

$Q_{e}=k_{F} C_{e}^{1 / n}$

where $Q_{m}(\mathrm{mg} / \mathrm{g})$ is the theoretical adsorption capacity of the fabrics, $k_{L}$ and $k_{F}(\mathrm{mg} / \mathrm{g})$ are the adsorption constants in Langmuir and Freundlich models, respectively. And $1 / n$ is a constant which indicates the strength of the adsorption between adsorbate and adsorbent.

The kinetics of the dyeing on Cotton and DEAE@Cotton were monitored by measuring the residual dye concentrations at different time intervals. The exhaustions (E\%) of DR23 on Cotton and DEAE@Cotton at equilibrium were calculated according to Eq. (6), which were used to demonstrate the dye adsorption rates of Cotton and DEAE@Cotton.

$E(\%)=\left(1-\frac{C}{C_{0}}\right) \times 100 \%$

where $C$ is the dye concentration in the dyeing bath at specific time interval, and $C_{O}$ is the initial concentration of dye in the dyeing bath.

\section{Results and discussion}

\section{Modification of DEAE@Cotton}

Given the unique chemical structure of DEAE-Cl possessing both nucleophilic (tertiary amine) and electrophilic $(\mathrm{R}-\mathrm{Cl})$ reactive sites, alkaline-activated cotton can be cationized through a nucleophilic substitution reaction on $\mathrm{R}-\mathrm{Cl}$ accompanied with the self-propagation of DEAE-Cl. Three reaction paths shown in Scheme 1 can introduce cationic moieties onto the cellulose structure (Tang et al. 2019, 2020), with the following properties provided on the fabrics: (1) covalently bonded cationic groups possessing excellent durability for dyeing, printing, and 
laundering; (2) strong affinity to anionic dyes in a saltfree dyeing system; (3) improved color fastness compared with conventional dyeing methods; and (4) retention of mechanical properties of treated cotton fabrics. To optimize the dyeability of DEAE@Cotton, critical modification parameters were carefully studied.

As shown in Fig. 1a-c, $\mathrm{NaOH}$ concentration in the activation step highly affected the final dyeability of
DEAE@Cotton since the alkaline condition not only could swell the cellulosic fibers to open more reactive sites to react with DEAE-Cl, but also ionize hydroxyl groups in cellulose structure to be more nucleophilic. By increasing the $\mathrm{NaOH}$ concentration from $0 \mathrm{~g} / \mathrm{L}$ (control group) to $60 \mathrm{~g} / \mathrm{L}$, the $\Delta \mathrm{E}$ of dyed fabrics over the conventional dyed Cotton $\left(L^{*}=48.07\right.$, $\left.a^{*}=54.17, b^{*}=24.88\right)$ decreased and $\mathrm{K} / \mathrm{S}$ values increased gradually. Nevertheless, the dyeability was
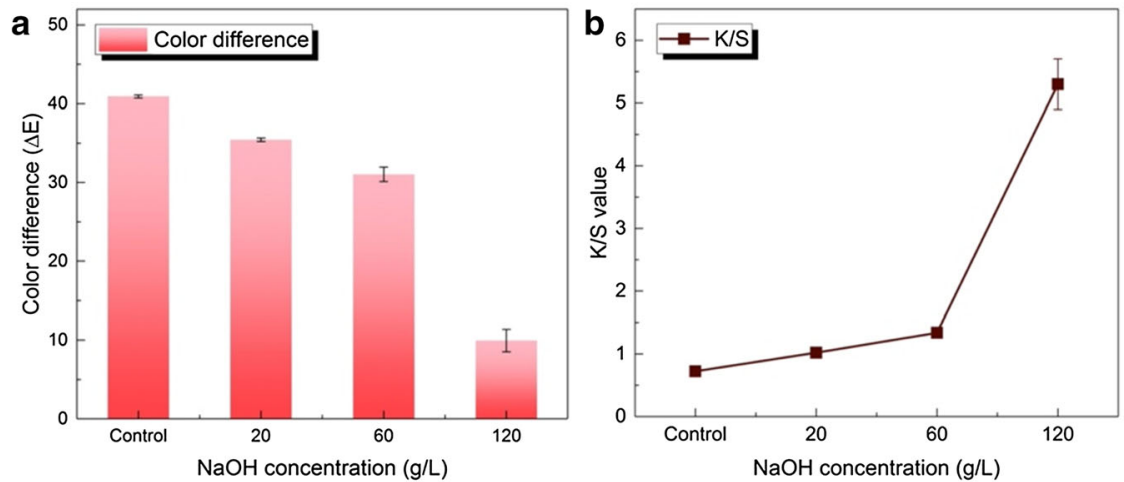

C
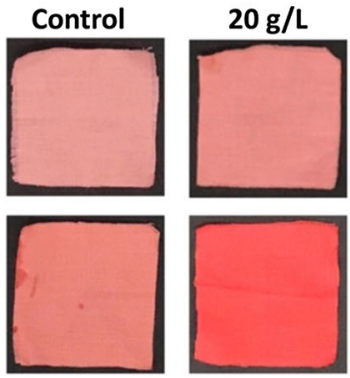

$60 \mathrm{~g} / \mathrm{L}$

$120 \mathrm{~g} / \mathrm{L}$
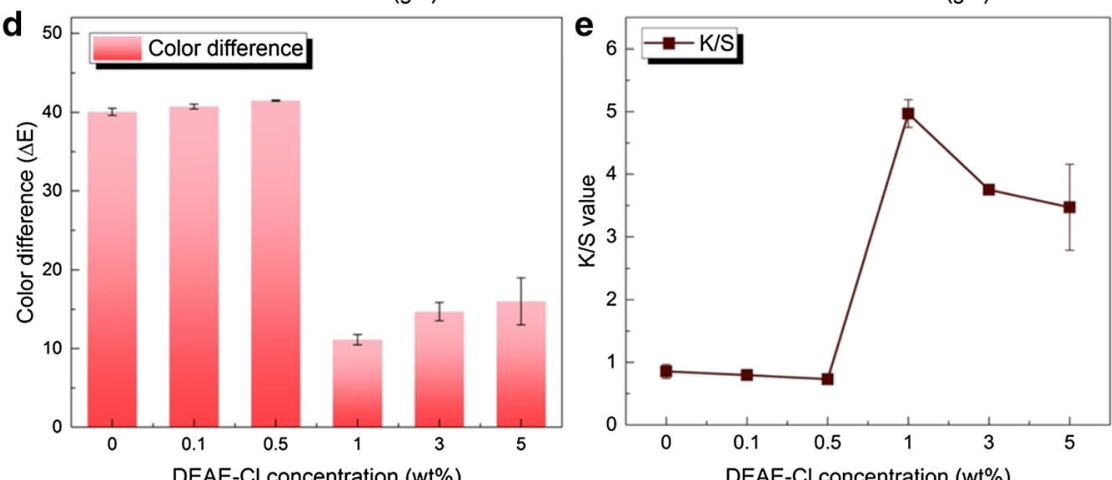

f
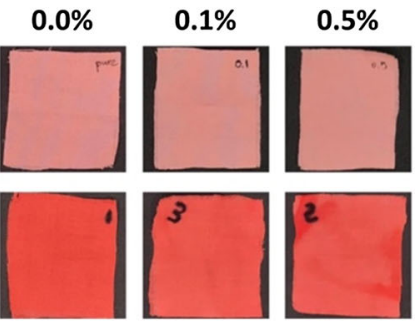

$1.0 \%$

$3.0 \%$

$5.0 \%$
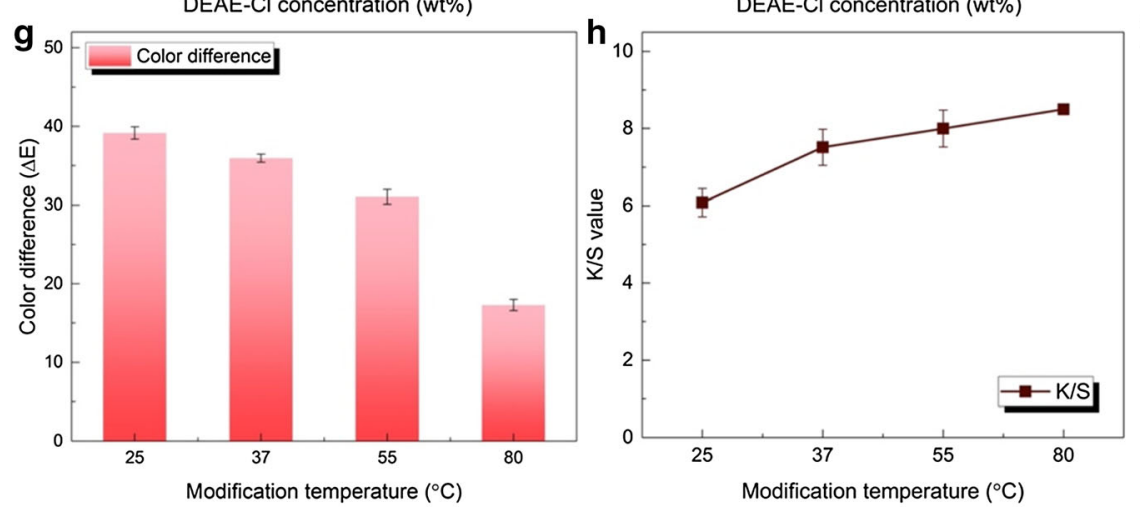

i

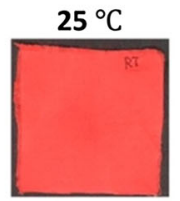

$37^{\circ} \mathrm{C}$

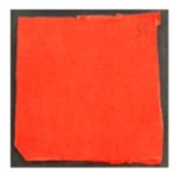

$55^{\circ} \mathrm{C}$

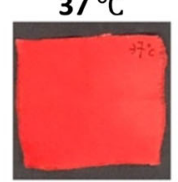

wt $\%$, and dyeing temperature of $25{ }^{\circ} \mathrm{C}$ were used in selection of each single variable. c, f, and i Optical images of dyeing performance of DEAE@Cotton fabrics obtained from different modification conditions

Fig. 1 a, d, and $\mathbf{g}$ Color differences $(\Delta \mathrm{E})$ and $\mathbf{b}$, e, and $\mathbf{h} \mathrm{K} / \mathrm{S}$ values of DEAE@Cotton fabrics modified with various $\mathrm{NaOH}$ concentrations, DEAE-Cl concentrations, and DEAE-Cl modification temperatures, respectively. Standard conditions with $\mathrm{NaOH}$ concentration of $120 \mathrm{~g} / \mathrm{L}$, DEAE-Cl concentration of 1.0 
improved significantly when cotton was pre-treated with $120 \mathrm{~g} / \mathrm{L} \mathrm{NaOH}$ by showing a $\Delta \mathrm{E}$ and $\mathrm{K} / \mathrm{S}$ value of 9.96 and 5.30 after a salt-free dyeing, which have been improved by 4.11 and 7.34 folds than that of the control group (i.e., Cotton dyed without salts), respectively (Fig. 1a, b). In fact, the alkaline activation step is similar to the mercerization process, which is a necessary preparation treatment of cotton fabrics during textile manufacturing.

The dyeability of DEAE@ Cotton can also be varied according to the DEAE-Cl concentration employed in the reaction system, because the DEAE-Cl concentration will determine the occurrence of different reactions shown in Scheme 1 and the number of cations introduced by the quaternized groups. The propagation of DEAE-Cl on the DEAE-grafted cotton fabric is crucial to maximize the cationization of the fabric with limited reactive sites in cellulose structure. As shown in Fig. 1d-f, the $\Delta \mathrm{E}$ of the dyed DEAE@Cotton compared to conventionally dyed Cotton decreased by increasing the DEAE-Cl concentration from 0 to 1.0 $\mathrm{wt} \%$ and increased slightly when the concentration reached $5.0 \mathrm{wt} \%$. In this case, the proposed reactions of (1), (3) and (4) are expected to coexist, maximizing the cationization of DEAE@Cotton. However, the further increment of DEAE-Cl concentration to 3.0 wt $\%$ or $5.0 \mathrm{wt} \%$ did not present better dyeing performance. This is caused by that the high DEAE$\mathrm{Cl}$ concentration could accelerate the self-propagation of DEAE-Cl (reaction 2) and compete with the propagation reaction on cotton (reaction 3). Selfpolymerized DEAE-Cl in reaction 2 has reduced reactivity with cellulose due to the steric hindrance to the $\mathrm{C}-\mathrm{Cl}$ site and lower the number of cations on the cotton fibers. Therefore, the optimal DEAE-Cl concentration was selected as $1.0 \mathrm{wt} \%$ for further studies.

The reaction temperature in the DEAE-Cl modification step has a slight impact on the dyeability of DEAE@Cotton, and the color intensity on the dyed fabrics moderately increased (i.e., the $\Delta \mathrm{E}$ changes oppositely) when raising the dyeing temperature from 25 to $80{ }^{\circ} \mathrm{C}$ (Fig. $1 \mathrm{~g}-\mathrm{i}$ ). It further emphasized the importance of high concentration of cationic groups on the DEAE@Cotton, providing strong attractive force to anionic dyes. With an overall consideration of chemicals usages and energy costs, the optimal modification condition for preparation of DEAE@Cotton was selected as $\mathrm{NaOH}$ concentration, DEAE- $\mathrm{Cl}$ concentration, and modification temperature of $120 \mathrm{~g} / \mathrm{L}, 1.0 \mathrm{wt} \%$, and $55{ }^{\circ} \mathrm{C}$, respectively in this study.

\section{Characterization of DEAE@Cotton}

Proposed chemical structure and properties of DEAE@Cotton were characterized and ascertained with SEM, FTIR, TGA, zeta potential measurements and wicking tests, as shown in Fig. 2. The incorporated cationic moieties on the DEAE@Cotton could highly improve the fabric dyeability according to an intermolecular electrostatic force shown in Fig. 2a. The positively charged quaternary ammonium groups facilitate the dye adsorption process by converting charge repulsion between Cotton and anionic dyes to an electrostatic attraction (i.e., the major interaction between DEAE@Cotton and anionic dyes). The SEM images of the cotton fabrics before and after DEAE-Cl modification present different fiber morphologies. The DEAE@Cotton revealed fibers with smoother surface but the fibers in Cotton were mostly in flat ribbonshape (Fig. 2b). However, an external force, which applied during the cotton mercerization, was not imposed here during the alkaline treatment, so the cross-sectional view of the DEAE-Cotton fiber only shows slight swelling effect instead of presenting a round shape (Fig. 2b). The $\mathrm{NaOH}$ solution could effectively swell cotton fibers, ensuring the DEAE-Cl penetration into the fibers and improving the modification effectiveness. As shown in Fig. 2c, the FTIR results proved the chemical structure difference between Cotton and DEAE@Cotton. A subtracted spectrum of Cotton from DEAE@Cotton clearly shows the decrement of hydroxyl group $(-\mathrm{OH})$ and the increase of methylene groups $\left(-\mathrm{CH}_{2}-\right)$ and ether groups $(\mathrm{C}-\mathrm{O}-\mathrm{C})$ in the DEAE@Cotton, which expressed as a negative absorbance at $3480 \mathrm{~cm}^{-1}$ and positive absorbances at $1490 \mathrm{~cm}^{-1}$ and $1261 \mathrm{~cm}^{-1}$, respectively (Tang et al. 2019, 2020; Yang et al. 2018). The changes of these characteristic peaks successfully demonstrated the formation of covalent linkages between cellulose and DEAE-Cl. Meanwhile, the different absorbance patterns around $2900 \mathrm{~cm}^{-1}$ presented the difference of saturated $\mathrm{C}-\mathrm{H}$ stretching between Cotton and DEAE@Cotton fabrics. The limited surface area of conventional cotton fabrics resulted in a relatively lower grafting ratio of DEAE moieties compared to that on surfaces of nanofibers (Tang et al. 2020). The DEAE moieties on 
a

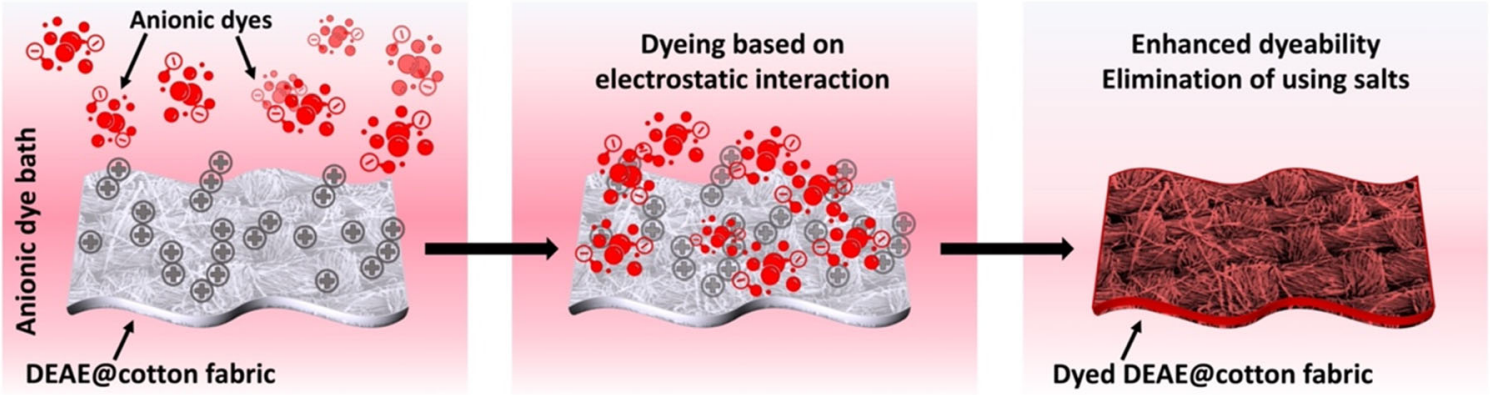

b

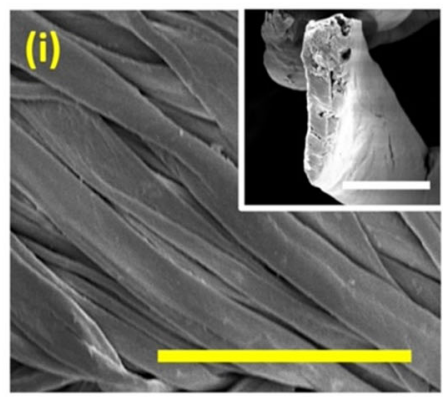

d

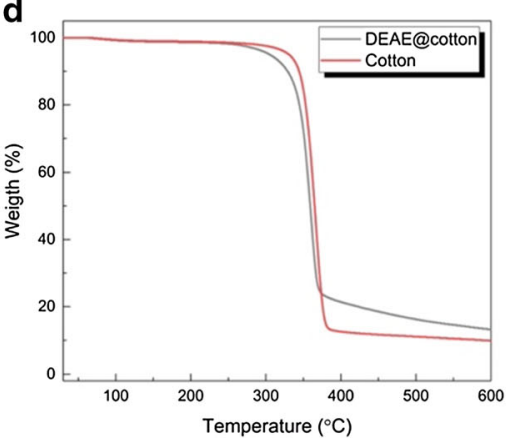

e

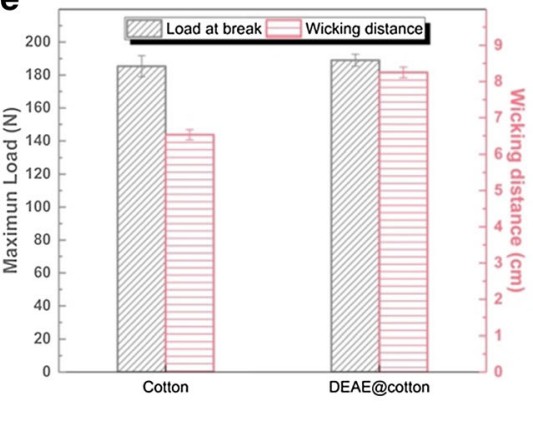

C
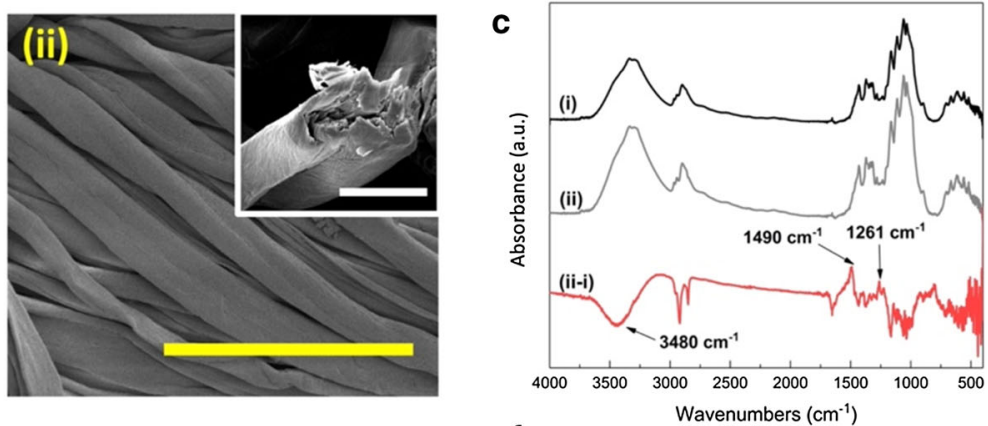

f

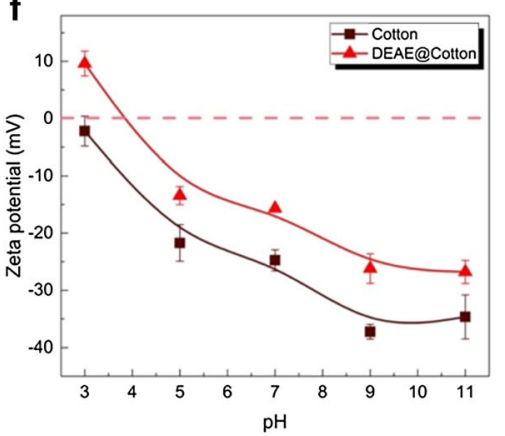

Fig. 2 a Scheme of enhanced dyeability of DEAE@Cotton fabrics with anionic dyes. b SEM images of (i) Cotton, and (ii) 1.0 wt\% DEAE@Cotton. The yellow bar refers to $100 \mu \mathrm{m}$. The white bar in the inserted figures stands for $10 \mu \mathrm{m}$. c FTIR results of (i) Cotton, (ii) 1.0 wt\% DEAE@ Cotton, and (ii-i) the subtract

the DEAE@Cotton are lack of distinguishable FTIR characteristic peaks, making a challenge to obviously notice the structural differences of the DEAE@Cotton over the pristine cotton. However, our previous studies by adopting the same chemistry for nanofibrous membrane cationizations have already structurally demonstrated the success of proposed reaction (Tang et al. 2019, 2020).

Moreover, the TGA results (Fig. 2d) showed higher thermal degradation residues of DEAE@Cotton at $600{ }^{\circ} \mathrm{C}$ compared with Cotton, which is attributed to the addition of DEAE moieties in DEAE@Cotton. On the other hand, the cationization of cotton fabrics with spectrum of (i) from (ii). d TGA results of Cotton and $1.0 \mathrm{wt} \%$ DEAE@Cotton fabrics. e Mechanical strength and wicking performance of Cotton and 1.0 wt\% DEAE@Cotton fabrics. f Zeta potentials of Cotton and 1.0wt\% DEAE@Cotton under different $\mathrm{pH}$ conditions

DEAE-Cl is expected to increase the hydrophilicity of treated fabrics by having additional cationic moieties in DEAE@Cotton. As shown in Fig. 2e, the wicking distance of DEAE@Cotton achieved $8.2 \mathrm{~cm}$ while the distance of Cotton was only $6.5 \mathrm{~cm}$. The tensile strength of DEAE@Cotton did not show any difference to the Cotton fabric, ensuring desired applications of DEAE@Cotton in textiles. On the other hand, the electrostatic charges (zeta potentials) of DEAE@Cotton under varied solution $\mathrm{pH}$ values were measured and compared with that of Cotton in aqueous system. The results are shown in Fig. $2 \mathrm{f}$. With increasing the solution $\mathrm{pH}$ values from 3 to 11 , 
the zeta potentials of Cotton and DEAE@Cotton both gradually decreased, which is caused by the deprotonation of the hydroxyl groups in cellulose by hydroxyl anions. Although DEAE@Cotton only showed a positive zeta potential under an acidic condition (i.e., $\mathrm{pH}<4$ ), the average zeta potentials of the DEAE@Cotton were constantly around $10 \mathrm{mV}$ higher than that of Cotton in the varied $\mathrm{pH}$ range (Fig. 2f), which is another evidence of the formation of cationic quaternary ammonium salts on the DEAE@Cotton. Since the zeta potential measurements were conducted in a fabric suspension, more negatively charged hydroxyl groups inside the fiber could expose in the suspension but were not accessible by the DEAE-Cl during the modification. The decrement of negative charges on the fabric surface weakened the charge repulsion toward anionic dyes and can eventually achieve better dyeability of DEAE@Cotton.
Dyeing performance on DEAE@Cotton

As shown in Fig. 3a-c, the dyeability of DEAE@Cotton with direct red 23 (DR23) without addition of inorganic salt is comparable to the Cotton dyed by the conventional method $(10 \mathrm{~g} / \mathrm{L} \mathrm{NaCl}$ in the dyeing bath). The $\Delta \mathrm{E}$ and $\mathrm{K} / \mathrm{S}$ value of the dyed fabrics are shown as 6.471 and 8.924, respectively (Fig. 3a, b). Moreover, the addition of different amount of salt for dyeing DEAE@Cotton did not show obvious improvement in color intensity. These results further demonstrated the efficiency of the cotton cationization reaction with DEAE-Cl and the effectiveness of using DEAE@Cotton to liberate the usage of inorganic salts during cotton dyeing.

The direct dyed cotton fabrics normally have low wash fastness. By introducing a strong electrostatic interaction between cotton fabric and direct dye, the wash fastness of DR23 dyed DEAE@Cotton was highly improved (Fig. 3d). The K/S values of DR23 dyed Cotton dropped dramatically from 10.13 to 7.94 after one wash, whereas the $\mathrm{K} / \mathrm{S}$ value of the
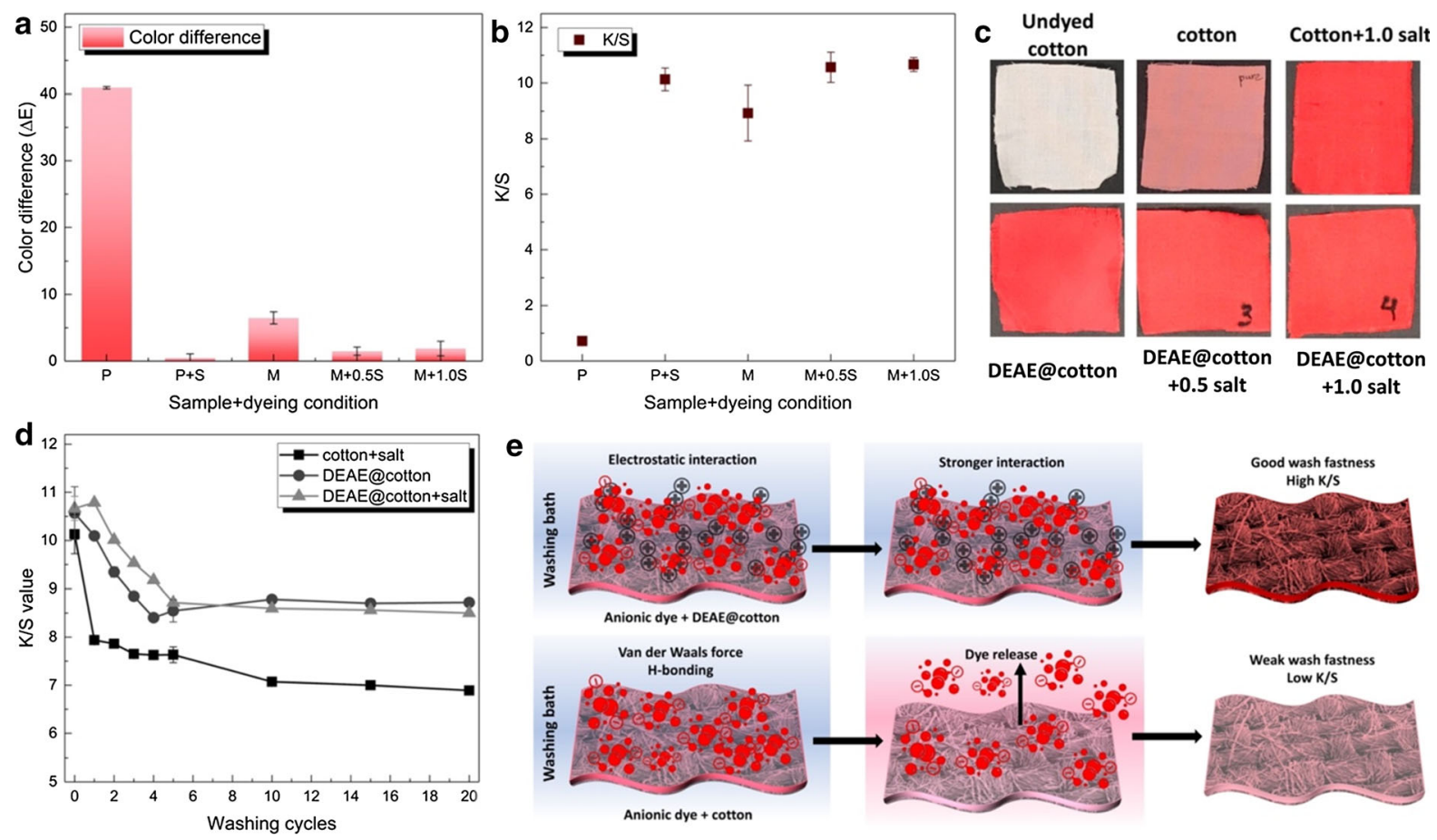

Fig. 3 a Color differences $(\Delta \mathrm{E})$ and $\mathbf{b} \mathrm{K} / \mathrm{S}$ values of dyed cotton fabrics under different conditions. The abbreviations of $\mathrm{P}$, $\mathrm{P}+\mathrm{S}, \mathrm{M}, \mathrm{M}+0.5 \mathrm{~S}$, and $\mathrm{M}+1.0 \mathrm{~S}$ stand for Cotton, Cotton with one unit of salt, 1.0 wt\% DEAE@Cotton, 1.0 wt $\%$ DEAE@Cotton with 0.5 unit of salt, and 1.0 wt $\%$
DEAE@Cotton with one unit of salt, respectively. c Optical images of dyed fabrics. d Wash fastness of dyed fabrics within 20 cycles. e Scheme of the mechanisms of wash fastness on Cotton and 1.0 wt\% DEAE@Cotton 
DEAE@Cotton dyed without salts only decreased by 0.48 units (changed from 10.57 to 10.09) (Fig. 3d). Furthermore, the K/S value of the dyed-DEAE@Cotton decreased gradually within the first four cycles of washing, and the color intensity remained constant at $\mathrm{K} / \mathrm{S}=8.62$ after 20 washings, which was $\sim 2$ units higher than that of the dyed-Cotton (Fig. 3d). The addition of salts in dyeing DEAE@Cotton presented slightly better wash fastness within the first five washing cycles. After 20 cycles of washing, however, it reached the similar color intensity $(\mathrm{K} / \mathrm{S}=8.52)$ as the one without addition of the salt (Fig. 3d). As shown in Fig. 3e, the presence of electrostatic interaction between DEAE@Cotton and DR23 not only accelerated the dye adsorption during the dyeing process, which resulted in higher color intensity on the fabric, but also reduced the dye desorption during the initial laundering, a sign of improved interactive force between dyes and the fabric. Overall, the incorporation of cationic DEAE moieties on cotton fabrics provided dual functions of eliminating the usage of salts in the dyeing process and enhancing the color fastness during daily laundering.

On the other hand, the lightfastness and crocking fastness of the dyed cotton products are critical factors to further evaluate the colorfastness of the DEAE@Cotton. After daylight irradiation for 3 and $8 \mathrm{~h}$, the DR23 dyed-DEAE@Cotton only showed a $\Delta \mathrm{E}$ value of $1.52 \pm 1.05$ and $1.92 \pm 0.08$, respectively. This color change is barely noticeable by naked eyes. Although using the cationized cotton for salt-free dyeing resulted in more dye molecules stayed on the fiber surfaces, the lack of photoactivity of DR23 and the increased distance between dyes and hydrogen donors (i.e., the cellulose back bone) by DEAE chains contributed to the good lightfastness of the dyed DEAE@Cotton fabrics. The crocking fastness of the dyed DEAE@Cotton fabrics was evaluated under dry and wet conditions according to AATCC Test Method 8-1996. The crocking fastness ratings were 5 (no color transfer to the reference cotton) and 3-3.5 (a slight color staining to the reference cotton) under dry and wet crocking, respectively.

Thermodynamics and kinetics of dyeing on DEAE@Cotton

Equilibrium adsorption isotherms of the dyeing processes were investigated to understand the dye adsorption mechanism and explicate the different dyeing properties of Cotton and DEAE@Cotton. Langmuir and Freundlich isotherm models were applied to describe the adsorptions of DR23 on the fabrics under equilibrium $(24 \mathrm{~h})$, and the results are shown in Fig. 4a, b and Table 1. Freundlich isotherm better described the dyeing process of Cotton with a higher correlation coefficient $\left(R^{2}=0.9976\right)$, whereas the dyeing process of DEAE@Cotton is more consistent with the Langmuir isotherm model $\left(\mathrm{R}^{2}=0.9667\right)$, consistent with the electrostatic monolayer adsorption of anionic dyes on the cationized cotton fibers. The large number of cationic quaternary ammonium salts on the DEAE@Cotton provided strong attractive force to anionic dye molecules, thereby enhancing the subsequent dyeing process.

In addition, DEAE@Cotton can completely exhaust the dye molecules in solutions and leave no residual dyes in the dyeing bath due to the strong electrostatic interaction when the initial dye concentration was lower than $50 \mathrm{mg} / \mathrm{L}$. And the exhaustions of $100 \mathrm{mg} / \mathrm{L}$ and $200 \mathrm{mg} / \mathrm{L}$ dyeing solutions reached $95.70 \%$ and $41.27 \%$, respectively, which are 4.86 and 3.09 times higher than that of the Cotton. The dyeing kinetics within the dyeing duration (40 $\mathrm{min}$ ) are shown in Fig. 4d. Nearly $50 \%$ of the dyes in the dyeing bath were exhausted by the DEAE@Cotton after 40 min dyeing, whereas less than $5.0 \%$ of the dyes were fixed onto the Cotton, which exacerbated burden to subsequent wastewater treatment. In this way, the improved dye exhaustion and adsorption kinetics of DEAE@Cotton further emphasized the advantage of introducing DEAE moieties onto cotton fabrics as a more environmentally friendly process.

Affinity of DEAE@Cotton with other anionic dyes

Possessing the cationic DEAE moieties, the DEAE@Cotton is expected to present an improved affinity to other anionic dyes under a salt-free condition as well. Therefore, four water-soluble anionic dyes that are classified in three different dye groups (direct dye, acid dye, and reactive dye) were used to dye Cotton and DEAE@Cotton (dye structures are shown in Fig. 5). The $\Delta \mathrm{E}$ and $\mathrm{K} / \mathrm{S}$ values of dyed fabrics were measured and displayed in Fig. 6. Not surprisingly, more intense colors and higher $\mathrm{K} / \mathrm{S}$ values were achieved on the DEAE@Cotton without exceptions. Specifically, the $\Delta \mathrm{E}$ between Cotton and 

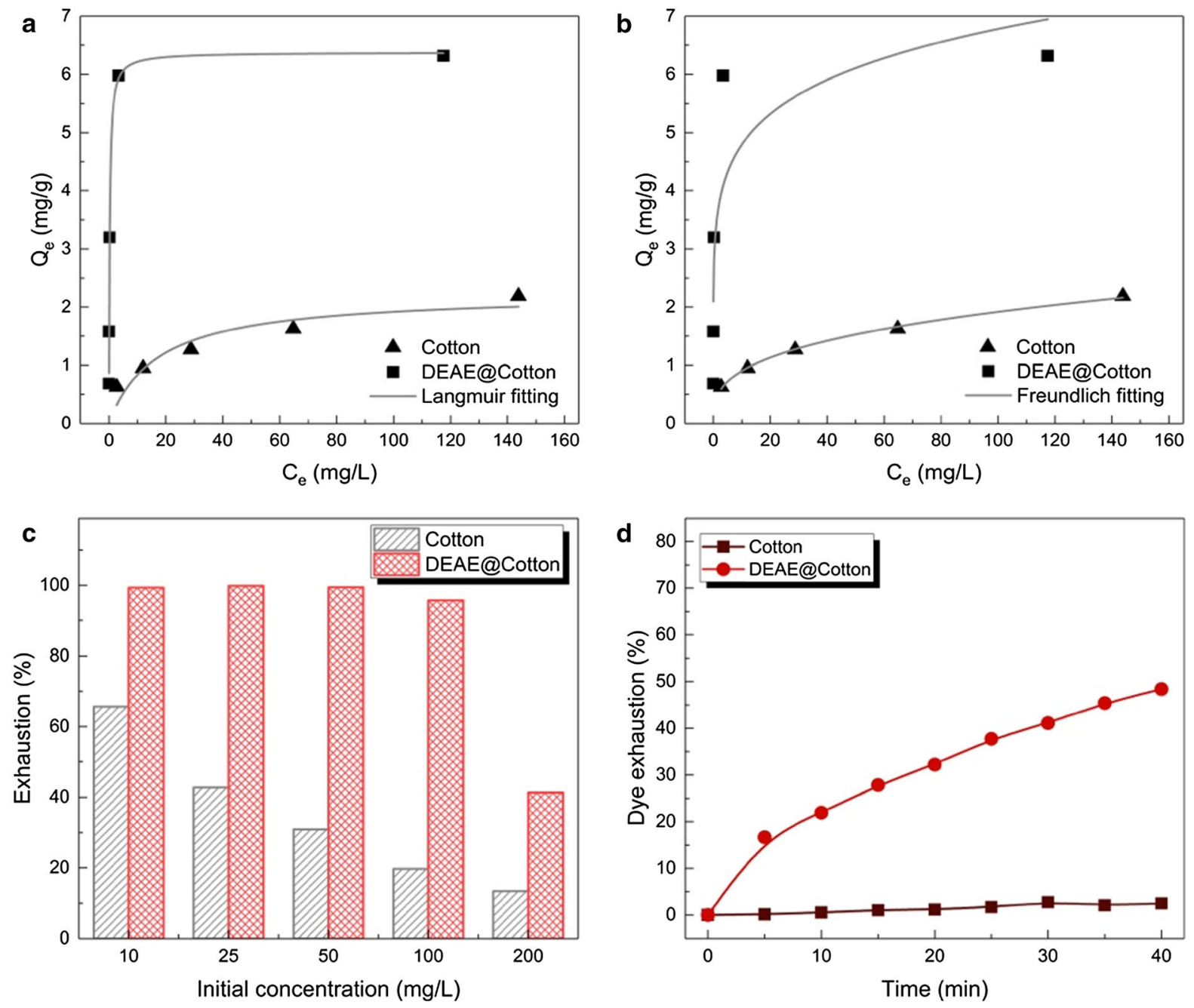

Fig. 4 Thermodynamic properties of DR23 adsorbed onto Cotton and DEAE@Cotton fabrics fitted with a Langmuir and b Freundlich isotherm models. c The dye exhaustion of Cotton and DEAE@Cotton fabrics under different dye initial

concentrations until the adsorption reached equilibrium. d Kinetic dye adsorption on Cotton and DEAE@Cotton fabrics at room temperature

Table 1 Parameters of Langmuir and Freundlich isotherm models

\begin{tabular}{llllllll}
\hline Isotherm model & \multicolumn{2}{l}{ Langmuir } & & \multicolumn{2}{l}{ Freundlich } \\
\cline { 2 - 4 } \cline { 6 - 8 } Fabrics & $k_{L}$ & $Q_{m}(\mathrm{mg} / \mathrm{g})$ & $R^{2}$ & & $k_{F}(\mathrm{mg} / \mathrm{g})$ & $n$ & $R^{2}$ \\
\hline Cotton & 0.059 & 2.239 & 0.8806 & 0.424 & 3.05 & 0.9976 \\
DEAE@Cotton & 3.825 & 6.376 & 0.9667 & 3.388 & 6.64 & 0.7317 \\
\hline
\end{tabular}

DEAE@Cotton dyed by DB71, DY27, AR151, and RB4 without adding salts are 24.53, 14.89, 17.82, and 21.10, respectively (Fig. 6a). Correspondingly, the $\mathrm{K} / \mathrm{S}$ values of the dyed-DEAE@Cotton fabrics showed 7.37, 4.00, 3.81, and 7.85 times higher than the cases that were dyed on Cotton without addition of salts (Fig. 6b). The optical images of dyed fabrics are shown in Fig. 6c. 
<smiles>COc1c(N=Nc2ccccc2)c(O)c2ccc(NC(=O)Nc3ccc4cc(N=Nc5ccc(NC(C)=O)cc5)c(S(=O)(=O)O)cc4c3)cc2c1C(C)(C)C</smiles>

Direct red 23

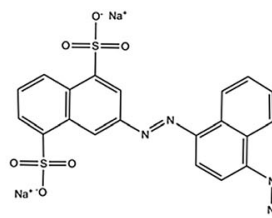

Direct blue 71
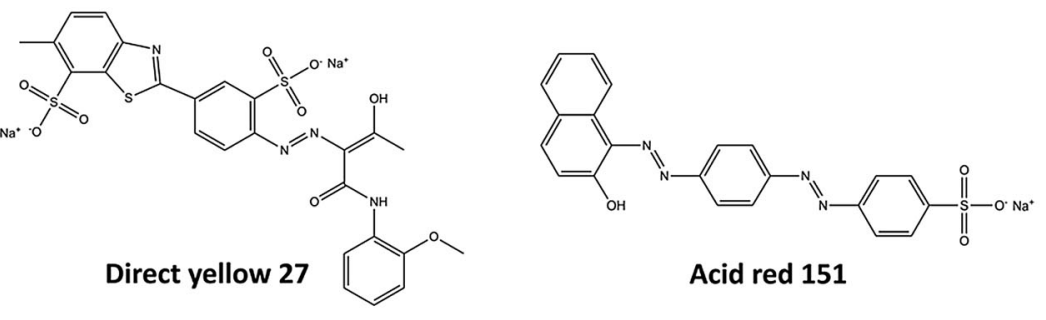

Acid red 151

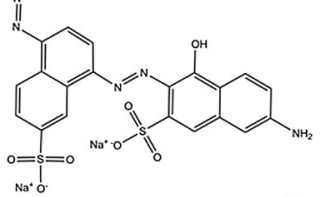

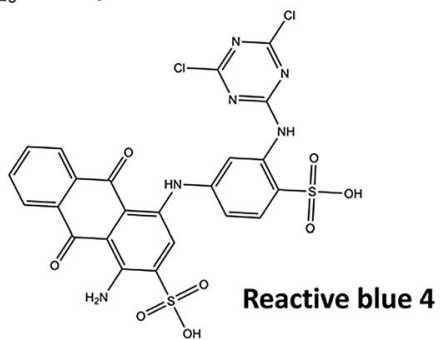

Fig. 5 Anionic dyes used in this study

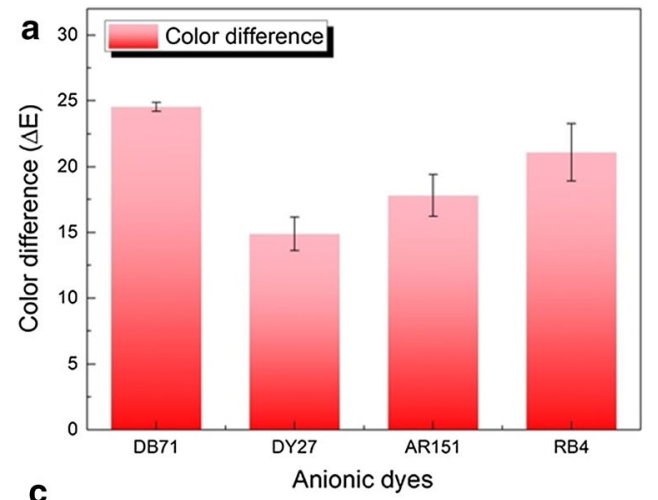

C
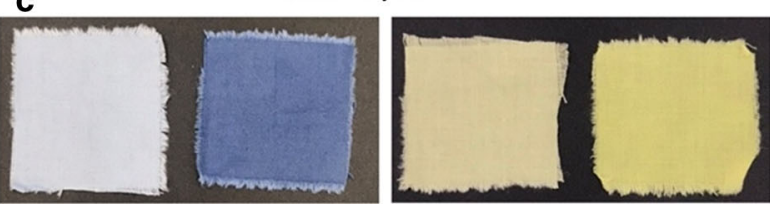

Fig. 6 a Color differences $(\Delta \mathrm{E})$ between dyed Cotton and dyed DEAE@Cotton and $\mathbf{b} \mathrm{K} / \mathrm{S}$ values of dyed fabrics with different anionic dyes. The labels in $\mathrm{x}$-axial refer to different dyes and cotton samples (C refers to cotton and M refers to DEAE@Cotton). c Optical images of dyes fabrics with direct blue 71

\section{Conclusion}

DEAE@Cotton fabrics were fabricated through a nucleophilic substitution reaction between hydroxyl groups in cotton and 2-diethylaminoethyl chloride (DEAE-Cl), followed by a DEAE-Cl propagation on the fabric. The coexistence of nucleophilic and electrophilic sites in DEAE-Cl is attributed to the
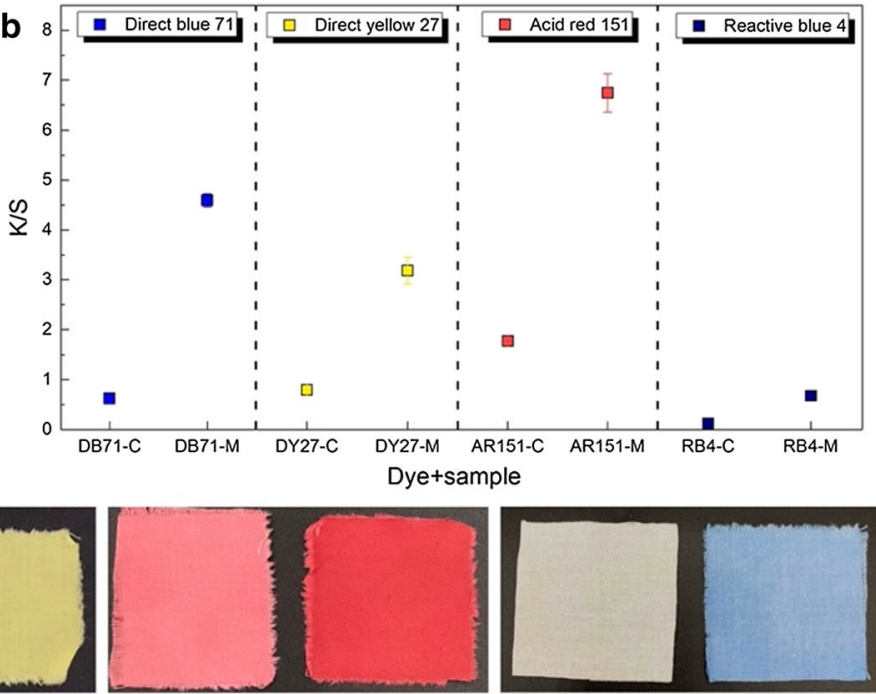

(DB71), direct yellow 27 (DY27), acid red 151 (AR151), and reactive blue 4 (RB4) (from left to right). Cotton are on the lefthand side and DEAE@Cotton are on the right-hand side

graft of tertiary amines onto cotton fabrics and a continuous growth of cationic quaternary ammonium salts, and eventually formed multiple cationic structures on one hydroxyl group. The proposed chemical structure of DEAE@Cotton was well demonstrated with FTIR, SEM, and TGA. DEAE@Cotton showed improved dyeability toward anionic dyes by introducing electrostatic interaction as the main force between 
direct dyes and the fabric. The dyeing performance was competitive to the color intensity on the Cotton that was dyed under conventional condition yet with much better color fastness against daily laundering. The cationization of cotton fabrics with DEAE-Cl successfully eliminated the application of inorganic salts in traditional cotton dyeing process, making the anionic dyeing of cotton fabrics more environmentally friendly.

Author contributions P. Tang: Conceptualization, Methodology, Validation, Writing-Original Draft; L. E. Lockett: Investigation, formal analysis; M. Zhang: Investigation; G. Sun: Conceptualization, Supervision, Writing-Review \& Editing.

Funding This work was partially supported by the California Department of Pesticide Regulation, USA (Grant No. 18-C0012), and the Jastro Shields Graduate Research Award at the University of California Davis, CA, USA.

\section{Declarations}

Conflict of interest Authors claim no conflict of interest.

Ethical approval No animal studies or human participants were involved in this study.

Open Access This article is licensed under a Creative Commons Attribution 4.0 International License, which permits use, sharing, adaptation, distribution and reproduction in any medium or format, as long as you give appropriate credit to the original author(s) and the source, provide a link to the Creative Commons licence, and indicate if changes were made. The images or other third party material in this article are included in the article's Creative Commons licence, unless indicated otherwise in a credit line to the material. If material is not included in the article's Creative Commons licence and your intended use is not permitted by statutory regulation or exceeds the permitted use, you will need to obtain permission directly from the copyright holder. To view a copy of this licence, visit http://creativecommons.org/licenses/by/4.0/.

\section{References}

Arivithamani N, Giri Dev VR (2017) Sustainable bulk scale cationization of cotton hosiery fabrics for salt-free reactive dyeing process. J Clean Prod 149:1188-1199

Dong X, Gu Z, Hang C et al (2019) Study on the salt-free lowalkaline reactive cotton dyeing in high concentration of ethanol in volume. J Clean Prod 226:316-323

Dixit S, Yadav A, Dwivedi PD, Das M (2015) Toxic hazards of leather industry and technologies to combat threat: A review. J Clean Prod 87:39-49
Fang K, Zhao H, Li J et al (2017) Salt-free dyeing of cotton fabrics modified with cationic copolymer nanospheres using an acid dye. Fibers Polym 18:400-406

Fang K, Xie R, Liu X et al (2019) Reactive dye/poly(styrene-cobutyl acrylate-co-trimethyl(vinylbenzyl) ammonium chloride) nanospheres with high coloration performance for cleaner dyeing of cotton fabrics. Cellulose 26:5807-5819

Farrell MJ, Ankeny MA, Hauser PJ (2014) Prediction of recipes for cotton cationisation and reactive dyeing to shade match conventionally dyed cotton. Color Technol 130(5):363-367

Farrell MJ, Fu S, Ankeny MA (2017) Cationic cotton prepared with hydrophobic alkyl chlorohydrin quats: a new fiber with new properties. AATCC 2017:97-125

Fu S, Hinks D, Hauser P, Ankeny M (2013) High efficiency ultra-deep dyeing of cotton via mercerization and cationization. Cellulose 20:3101-3110

Hashem M, Hauser PJ, Smith B (2003) Reaction efficiency for cellulose cationization using 3-chloro-2- hydroxypropyl trimethyl ammonium chloride. Text Res J 73:1017-1023

Hauser PJ, Tabba AH (2001) Improving the environmental and economic aspects of cotton dyeing using a cationised cotton. Color Technol 117:282-288

Kant R (2012) Textile dyeing industry an environmental hazard. Nat Sci 04:22-26

Khatri A, Peerzada MH, Mohsin M, White M (2015) A review on developments in dyeing cotton fabrics with reactive dyes for reducing effluent pollution. J Clean Prod 87:50-57

Ma W, Meng M, Yan S, Zhang S (2016) Salt-free reactive dyeing of betaine-modified cationic cotton fabrics with enhanced dye fixation. Chinese J Chem Eng 24:175-179

Ma W, Shen K, Xiang N, Zhang S (2017) Combinative scouring, bleaching, and cationization pretreatment of greige knitted cotton fabrics for facilely achieving salt-free reactive dyeing. Molecules 22:2235

Mu B, Li W, Xu H et al (2019) Salt-free and environmentfriendly reactive dyeing of cotton in cottonseed oil/water system. Cellulose 26:6379-6391

Sadeghi-Kiakhani M, Safapour S (2018) Salt-free dyeing of cotton fabric modified with prepared chitosan-poly (propylene) imine dendrimer using direct dyes. Prog Color Color Coatings 11:21-32

Samanta AK, Kar TR, Mukhopadhyay A et al (2015) Studies on dyeing process variables for salt free reactive dyeing of glycine modified cationized cotton muslin fabric. J Inst Eng Ser E 96:31-44

Samanta AK, Kar TR, Mukhopadhyay A et al (2016) Ecofriendly salt-free reactive dyeing of cotton (muslin) fabric after cationization with amino acid from soya. Text Res J 86:2179-2192

Tang P, Nguyen NTH, Lo JG, Sun G (2019) Colorimetric detection of carcinogenic alkylating fumigants on a nylon 6 nanofibrous membrane. Part II: self-catalysis of 2-diethylaminoethyl-modified sensor matrix for improvement of sensitivity. ACS Appl Mater Interfaces 11:13632-13641

Tang P, Zhang M, Robinson H, Sun G (2020) Fabrication of robust functional poly-cationic nanodots on surfaces of nucleophilic nanofibrous membrane. Appl Surf Sci 528:146587 
Toprak T, Anis P, Kutlu E, Kara A (2018) Effect of chemical modification with 4-vinylpyridine on dyeing of cotton fabric with reactive dyestuff. Cellulose 25:6793-6809

Wang GW, Zheng CL, Sun J (2016) Synthesis and salt-free dyeing characteristics of cationic reactive dyes containing polyetheramine segments. Color Technol 132:344-349

Wang J, Gao Y, Zhu L et al (2018) Dyeing property and adsorption kinetics of reactive dyes for cotton textiles in salt-free non-aqueous dyeing systems. Polymers (basel) 10:1030

Xia L, Wang A, Zhang C et al (2018) Environmentally friendly dyeing of cotton in an ethanol-water mixture with excellent exhaustion. Green Chem 20:4473-4483

Xiao H, Zhao T, Li CH, Li MY (2017) Eco-friendly approaches for dyeing multiple type of fabrics with cationic reactive dyes. J Clean Prod 165:1499-1507

Yang X, Zhao Y, Li R et al (2018) A modified kinetic analysis method of cellulose pyrolysis based on TG-FTIR technique. Thermochim Acta 665:20-27

Zhang Y, Zhang W (2015) Clean dyeing of cotton fiber using a novel nicotinic acid quaternary triazine cationic reactive dye: Salt-free, alkali-free, and non-toxic by-product. Clean Technol Environ Policy 17:563-569

Zhang F, Chen Y, Lin H, Lu Y (2007) Synthesis of an aminoterminated hyperbranched polymer and its application in reactive dyeing on cotton as a salt-free dyeing auxiliary. Color Technol 123:351-357

Zhao J, Agaba A, Sui X et al (2018) A heterogeneous binary solvent system for recyclable reactive dyeing of cotton fabrics. Cellulose 25:7381-7392

Zheng C, Yuan A, Wang H, Sun J (2012) Dyeing properties of novel electrolyte-free reactive dyes on cotton fibre. Color Technol 128:204-207

Zhu Z, Wu P, Liu G et al (2017) Ultrahigh adsorption capacity of anionic dyes with sharp selectivity through the cationic charged hybrid nanofibrous membranes. Chem Eng J 313:957-966

Publisher's Note Springer Nature remains neutral with regard to jurisdictional claims in published maps and institutional affiliations. 